\title{
FLASH INTERVIEWS with a DCP near you
}

\section{Debbie Chandler}

Debbie is a 50-year-old lead dental nurse in special care dentistry in Dorchester. Debbie is married to Damian, an NHS administrator, and has three step-daughters: Hayley, Vicky and Zoe; and four grandchildren: Bethany, Noah, Jack and Layla.

How long have you worked in dentistry? Thirty-four years

Why did you choose dentistry for your career? From a careers talk on dental nursing at school.

Do you have any special responsibilities within your workplace? I split my time working in the clinical setting for routine clinics, sedation clinics and GA lists. I manage the nurses in the department and work alongside the department co-ordinator to ensure staffing levels are met and clinics, $\mathrm{GA} /$ sedation lists are put together and the domiciliary service is running correctly.

What do you like best about your job? The service we provide is very diverse and because of this no two days are the same.

What's the most challenging part of your job? Working within the team and liaising with community support teams to ensure that dental care is delivered in a way that best meets the needs of the patients without delay: this can on occasion be very challenging.

What are your outstanding ambitions? I am always looking for new challenges. What do you like to do outside work? Going to concerts with my husband and visiting fancy restaurants.

Tell us a secret. I LOVE Robbie Williams (although I don't think that is a secret with my colleagues!)

What do you like about BDJ Team? It gives me all the latest updates straight to my PC.

What three things could you not live without (besides people)? My convertible car, iPad and Robbie Williams.

What is the most challenging part of your job? Trying to fit everything in and make time for emergencies - most days we have two or three CERECs each taking up to two hours to prepare, design, glaze and fit so can often run late.

What are your outstanding ambitions? To be able to do my job until I retire - and the rate dentistry is progressing it seems unlikely I will still have the energy to keep up!

What do you like to do outside work? I like to go to a few exercise classes in between organising my children's busy schedules. I also enjoy DIY projects at home.

Tell us a secret. I used to get mistaken for Lorraine Kelly - a lot!

\section{Deborah Ryan}

Deborah Ryan

is a dental

therapist

in private

practice and

the community

dental service

in London. She

is very happily

married to

Robert Ryan, a

world-famous

author (well

quite a bit famous). They have three children called Bella, Gina and Gabz.

How long have you worked in dentistry? A long time, first as a dental nurse, then dental technician, then after three pregnant pauses, I retrained as a dental therapist.

Why did you choose dentistry for your career? Because folk need us, even when they don't realise they do!

Do you have any special responsibilities within your dental practice? Telling folk that they really need to see us regularly.

What do you like best about your job? When said folk listen and realise they have improved health and comfort.

What is the most challenging part of your job? Apart from the demands of CQC and the GDC? Saying the same thing over and over (but I don't mind if folk listen - see above).

What are your outstanding ambitions? To be a busy dental therapist IN PRACTICE (hello - any dentists out there?!)

What do you like to do outside work? Be on a horse.

Tell us a secret. I love lifting big chunks of calculus.

What three things could you not live without (besides people)? Chocolate (I know it's cariogenic), horses and warm boots in the winter. responsible for making sure the GP17s are completed and sent and payment schedules checked in my receptionist role, and staining and glazing CEREC restorations in my dental nursing role - that and watering the bay trees in the garden!

What do you like best about your job? The satisfaction of seeing patients' delight when they have their new CEREC crowns/inlays/ veneers fitted, knowing that I was part of their production.
What do you like about BDJ Team? The free online CPD is informative and accessible for all, and I also enjoy the interviews with other DCPs.

What three things could you not live without (besides people)? Hand cream, my woolly hat (in all weather), and my iPhone (according to my daughter).
TAKE PART If you would like to appear in a FLASH INTERVIEW, just email bdjteam@nature.com and include a digital photo of yourself.

bdjteam2014111 